\title{
Revenue sharing in semiconductor industry supply chain: Cooperative game theoretic approach
}

\author{
BIKRAM K BAHINIPATI*, ARUN KANDA and S G DESHMUKH \\ Department of Mechanical Engineering, Indian Institute of Technology Delhi, \\ Hauz Khas, New Delhi 110016 \\ *e-mail: bikrambahinipati@yahoo.co.in
}

MS received 7 March 2008; revised 13 October 2008

\begin{abstract}
This paper defines cooperation as the process of coordinating the objectives and activities of supply chain (SC) members. It also focuses on cooperation as a solution for hybrid coordination mechanism to form the basis for semiconductor industry supply chain management. In the complex and competitive environment of semiconductor industry supply chain, independent system members are facing the difficult task of providing/sharing incentives resulting from e-market activities in a fair and equitable manner. So, various other activities are necessary for the e-market to make revenue sharing operations more stable and reliable. In this context, the importance of coalition in enhancing the e-market capability, for revenue generation and sharing, is used to develop a possible mechanism for financial compensation to the supply chain members. Interpreting the supply chain as cooperation, the concepts of the Shapely value are used in this paper for analysing the revenue sharing problem. The motivation behind such a scheme is to align the supply chain members' cost structure with the bidding value during auction and bargaining for e-procurement. The appropriateness of the Shapely value is verified to ensure that a stable solution exists. The practical implication of this paper is how to make right decisions about revenue sharing. The principal contribution of this approach is for establishing a pooling coalition in order to provide a stable and cooperative solution.
\end{abstract}

Keywords. Supply chain coordination; revenue sharing; Game theory, Shapely value.

\section{Introduction}

Supply chain management (SCM) in semiconductor industry has a significant potential in creating competitive advantage by coordinating information, cash and material flow among the members (Chopra \& Meind 2004) and through efficiency improvement due to an increase in e-market responsiveness (Fisher 1997). The scope for utilizing e-market mechanisms,

*For correspondence 
where many suppliers and buyers act on real-time demand and supply of a product, becomes larger through advanced IT. These more efficient, electronic markets (EM) provide many opportunities for matching buyers and suppliers, aggregation or pooling effects which serve to reduce demand opportunities, inventory needs and production efficiencies, in addition to reducing lead times and stockouts, and improving customer service level (Park \& Suresh 2005).

Agency theory has emerged as the dominant theory in explaining the relationship in e-market places in the literature. The principal-agent relationship arises when a firm identifies and engages another firm for funding of a new venture, wherein problem may arise between the firms as a result of incongruent goals and potentially different risk preferences (Eisenhardt 1989). This theory assumes that all the firms are self-interested and boundedly rational, which gives rise to information asymmetry (Sauvee 1998). As a result, individual utility-maximizing behaviour is likely to emerge if proper incentives and controls to align the goals of the firms are not employed. Multi-agent system fails when heterogeneous items are auctioned in the e-market and the agents' valuation of these items are not additive. The major limitation of the agency theory approach for revenue sharing in the semiconductor industry supply chain (SSC) emanates from the idea of goal congruence between the partnering organizations. For collaborative environment, the researchers should not ignore the individual firm and his goals and vision, and should not place emphasis only on the major player in the market.

Current research defines cooperation as the process of coordinating the objectives and activities of the supply chain (SC) members (Premkumar 2003). The cooperation is driven by trust and commitment (Handfield \& Bechtel 2002). In the complex and competitive environment of semiconductor industry supply chain, the generations of revenue resulting from e-market activities are different in principle from the ones used in the traditional supply network planning, possibly reducing the profit margins. Treatment of fixed cost in e-market is not perfect in any form of market design. Semiconductor industries do not know in advance how many units of their products/components will be ordered when they are submitting the bids. As a result, it is difficult for them to establish the average offer price during each period. This may potentially cause inefficient supply or e-market instabilities. So, various other activities are necessary for the e-market to support revenue sharing operation more stable and reliable. However, fundamental conditions must be satisfied for cooperation based on trust and commitment for member organizations not to mislead this objective through opportunistic behaviour. E-market provides the most ideal coordination/collaboration structure to limit this scope.

The objectives of the current research are two fold: (a) design a general multi-lateral negotiation procedure concerning fair cooperative acceptance criterion for supply chain collaboration, and (b) explore the relationship between the stability of cooperation structures and revenue sharing structure of the game of link formation, analyse the importance of coalition in enhancing e-market capability for revenue generation, and developing a possible mechanism for financial compensation through revenue sharing to supply chain members.

A typical approach would be to allocate revenue based on fractional or proportional rule among all the members of the grand coalition in the pool market. The fractional rule assigns to every member a pre-determined fraction of the total revenue generated by the grand coalition. The fractional rule satisfies aggregate monotonicity and coalitional monotonicity (Mecca et al 2004; Plambeck \& Taylor 2004). Aggregate monotonicity implies that a member's share does not decrease if the value of the grand coalition increases marginally, while the value of all other coalitions remains fixed. The coalitional monotonicity implies that a member's share should not decrease if the values of all coalitions that contain the member increase marginally, while 
the value of remaining coalitions remains the same. This approach is easy to implement and has been used by many industries. However, the proportional allocation of revenue does not align with the 'member revenue generation potential' principle. Further, it may not be acceptable to e-market participants. Common revenue allocation faces the fundamental issue that there is often no mutually acceptable solution, which is much inferior to the best solution. There does not seem to be a great deal of attention paid to this issue, even though common revenue sharing approaches have been discussed in the context of traditional markets (Cachon \& Lariviere 2005). While the revenue allocation schemes often rely on engineering intuitions, a few of them are based on axiomatic framework of cooperative game theory.

The revenue allocation problem may be formalized as follows: Allocate the total revenue generated due to coalition among all members such that the allocation possesses certain desirable attributes, such as stability and equity. Stability of allocation means that no sub-coalition has any incentive to leave the grand coalition, while equity means that no sub-coalition subsidizes any other sub-coalition. The guiding principles of these desirable properties are qualitative and hence not unique.

The objective of the revenue sharing is to identify the e-market participants who are responsible for generating this revenue. In many real-world situations of semiconductor industry supply chains, identifying the revenue generating members can be difficult. So, we propose axiomatic approach for this problem. An axiomatic approach does not address the problem of identifying the revenue generating members directly; rather it develops a revenue sharing scheme that satisfies certain desired properties called axioms. To illustrate the problem of revenue allocation, a semiconductor industry supply chain in e-market for a single period case is considered.

Generally, it is not a straightforward exercise to evaluate the worth and contribution of each coalition in the semiconductor industry supply chain. This is due to the complexity and difficulty associated with relating the performance of a coalition to the revenue generated from the coordinated SC. A part of the complexity arises from the manner in which the system performance is measured and valued. To address this issue, a game theoretic approach is employed. It is important to emphasize that the present study does not propose an e-market for coalition in semiconductor industry supply chain, but rather concentrates on proposing a revenue sharing mechanism to the coalition members, to motivate them to participate in a cooperative environment.

The proposed theoretical framework for revenue allocation relies on an alternative market design based on coalition structures in semiconductor supply chain. An attractive feature of this model is that it enables a coalition member to better align its offers in e-market with the revenue generation potential. This is an important aspect that improves the acceptability of this design by market participants, which can be suggested in the standard e-market design for semiconductor industry supply chains. The axiomatic approach of the proposed game theoretic framework has desirable properties that improve the acceptability of the solution in an e-market environment.

The paper is organized as follows. Section 2 deals with a brief review of the applications of cooperative game theory in supply networks. Section 3 proposes a theoretical framework for negotiation between collaborating members prior to the development of a revenue sharing scheme based on cooperative game theory. Section 4 proposes a revenue generation model for supply chain coalition in e-market. Section 5 analyses the revenue sharing problem through Shapely approach and presents the observations from such analysis as well as the simulation results. Finally, Section 6 presents the concluding remarks of the present work. 


\section{Cooperative game theory}

The evolution of SCM identifies that a business process consists of several decentralized firms, and operational decisions of these firms impact each others' profit and the profit of the supply chain. In order to effectively model and analyse decision-making in such multi-member situation where the outcome depends on the choice made by each member, game theory is an obvious choice. Researchers in SCM now use tools from game theory and economics to understand, predict and help managers to make strategic operational decisions in complex multi-member SC systems.

Game theory deals with the modelling aspects of cooperation and conflict between the members of the supply chain to contribute towards mutual competitive advantage (Fundenberg \& Tirole 2005). Game theory models situations where the SC members make decisions to maximize their own utility, while taking into account that other members are doing the same and that decisions made by SC members impact each other's utilities. Even though game theory has been widely used in the supply chain literature, there is still a lack of concepts while analysing the supply chains in the area of revenue sharing for coordination/collaboration. The cooperative game theory directly looks at the set of possible outcomes, studies what the members can achieve, what coalitions will form, how the coalitions share the outcomes and whether the outcomes are stable and robust. The use of cooperative game theory is, however, much less prevalent. We review the existing literature on applications of cooperative games to supply chain management. Cooperative game theory has potential in supply chain management applications as cooperation is a driver for improved supply chain performance (Cachon \& Netessine 2004). Cooperative game theory has not been considered from a strategic perspective. There is a need for cooperative game-theoretic approach, where the members can cooperate with each other based on mutual understanding and agreements in order to have a stable joint outcome.

There are two central themes of cooperative games (Nagarajan \& Sosic 2006). The first theme is that of the feasible outcomes. Feasible outcomes represent the total set of all possible outcomes that the members may realize, even if they may not be incentive compatible for them. Identifying this set and its properties is an important step. Once this set is established and defined, it is essential to analyse how the members actually end up with an outcome from this feasible set. Cooperative game theory offers several applications of this process, one important application being the bargaining between the players. The second important theme is that of stability. When the SC members decide on allocations from the set of feasible outcomes independent of the process, some or all members can opt for joining together as a coalition and agreeing on a joint course of action. So, the problems arise as to how the members in an alliance share the gains generated by their joint venture and what stable conditions or outcomes that emerges in a particular setting.

Coalition have been accepted and recognized as the essential means for stable and secure system of revenue sharing for semiconductor industry supply chain (Greene 2002; Cachon \& Lariviere 2005). This approach renders a service to the SC system by providing damping action when the system disturbances influence the reliability of the revenue sharing among the members. Therefore, it is reasonable to argue that coalitions in e-market should be considered as an ancillary service provider (Park \& Suresh 2005). A secured revenue sharing system is critical for efficient e-market operation, which plays an important role in revenue generation and capability limits of members of semiconductor industry supply chain. The amount of allowable revenue transactions in e-markets needs to be evaluated to ensure operational feasibility and motivation among these SC members. The revenue sharing cost analysis is 
carried out using a risk-taking strategy that provides proper e-market signals to the participating members in the market. It is essential to examine in detail the importance of each single coalition in the semiconductor industry supply chain and the extent to which it contributes to the stabilization of the revenue sharing systems (Plambeck \& Taylor 2004).

The aspects of feasible outcomes and stability are closely related to revenue sharing in cooperation. So, there is a need to explore the cooperative game theory due to its inherent potential to provide a rationality-based foundation for developing algorithms for revenue sharing. These algorithms must be designed for stable solutions, which must depend on the profit contributed by individual members in coalition. Coalition theory analyses the scenarios in which the participating members commit to specific strategy due to the existence of mechanisms, such as purchasing agreements or contracts. So, cooperative behaviour among supply chain members is assumed for effective negotiation.

While there exist a significant body of work dealing with coalition formation and stability in economics literature (Greenberg 1994), there is very little research done within this framework in the SCM literature. SCM gives importance to the cooperation among the members in the supply chain in order to improve the performance. However, only a few researchers so far have tried to use models from cooperative game theory, which appears to be a natural framework for such analysis. Cachon \& Netessine (2004) provide a brief (but extensive) literature survey of papers in SCM that adopt cooperative concepts (the core, and the Shapely value).

\subsection{Full cooperation structure and contractual stability}

This paper attempts at developing a theoretical framework that allows us to study which bilateral links and coalition structures are going to emerge at equilibrium. This new framework would enable us to redefine the revenue sharing (RS) rules and to introduce a new solution concept, i.e. contractual stability.

Intuitively, if two SC members do not form a link, it is as if they commit to refuse direct cooperation, but they do not rule out the possibility of cooperation through e-marketplace. If the game is superadditive, then the full cooperation structure (each member cooperates with all the other members) is stable in the sense that no member can profitably deviate by serving his links or any part thereof (Quin 1996). In the full cooperation structure, all the SC members are linked between each other. The motivations for studying this structure are: (a) In RS gaming situations, the full cooperation structure is the efficient one and the SC managers would like it to be a stable one. (b) It has an interesting property of making a bridge between networks and coalitions. From a network point of view, a coalition can be seen as a network where all the SC members are linked to each other.

It is shown in the literature that the full cooperation structure is stable, if and only if the Shapely value of each member is individually rational and the RS game is superadditive for coalitions (Haeringer 2001). The definition of superadditivity is as follows: If $S$ and $T$ are any coalitions of a game, and $S \cap T=\phi$, then the game is superadditive if

$$
P(S)+P(T) \leq P(S \cup T) .
$$

This suggests that small coalitions have a greater role in the stability of full cooperation structure than bigger ones. In fact, all coalitions do matter since whether or not the Shapely value (Shapely 1953) of each player is individually rational depends on the profit of all coalitions. This result also shows that the underlying game does not need to be superadditive to ensure stability of the full cooperation structure. This is an interesting property given that nonsuperadditive economic environments are common in semiconductor industry supply chain. 
Such environments occur when there are congestion effects in the manufacturing of components or products or when there are inefficiencies in decision making in large semiconductor manufacturing industries. In such large firms or organizations, increasing return to scale can be dominated by cost coordination (Bela \& Goyal 2000).

Aumann \& Myerson (1988) suggested a model for the endogenous formation of cooperation structures. By allowing SC members to strategically form or to refuse links, they wished to study the nature of cooperation structure that will emerge under the assumption that payoffs are determined by Myerson value (the Shapely value of the game induced by cooperation structure (Myerson 1977). The stable structure has three properties (Feinberg 1998):

(i) It is connected (every pair of SC members is connected through a chain of links) and is incomplete (not all links are present).

(ii) It is stable under every protocol (no new links will be added strategically to the cooperation structure under the restriction to the sub-game perfect equilibria in a linking game with an arbitrary protocol).

(iii) When new link is offered to two SC members, at least one of them will refuse it. The reason being that the refusing party could expect a decrease in its strength (profit sharing), if that link is added to the cooperation structure due to the triggering of the addition of other links.

The idea of contractual stability is that adding or deleting a link needs the consent of coalition partners. In the context of R\&D alliance in the semiconductor industry supply chain, the firms may decide to have a common laboratory with some partners, while developing bilateral R\&D agreements with other partners. The signing of a bilateral R\&D agreement may need the consent of those partners with the common laboratory or joint venture. Moreover, the formation of new coalition structures needs the consent of original coalition partners. Thus, once a coalition has been formed, the consent of all coalition partners is considered in order to modify the existing coalition that affects some coalition partners. A simple way to analyse the coalition networks that one might expect to emerge in the long run is to examine a sort of equilibrium requirement that no coalition benefits from altering the coalition network (Caulier et al 2007). Goyal \& Joshi (2003) have analysed the incentives for R\&D collaboration between horizontally related firms by considering that collaboration links are bilateral and are embedded within a broader network of similar links with other firms. Goyal \& Joshi (2006) have also investigated the formation of free trade agreements as a network formation game.

\subsection{Rationality axioms and allocations based on core}

The best known stability concepts are the core (Gillies 1959) and the coalition structure core (Aumann \& Dreze 1974). The core possesses a stability property — when core allocations are used, no sub-set of members has an incentive to leave from the grand coalition and form its own coalition. Its main drawback is that, in general, it can be an empty set. While the core addresses the stability of the grand coalition, the coalition structure core addresses the stability of an arbitrary coalition structure. The SCM literature is seemed to be interested only in the stability of the grand coalition. Leng \& Parlar (2005) showed the non-emptiness of the core for the information sharing game.

Assume $X_{A}, X_{B}$, and $X_{C}$ to be the revenue allocated to the coalition members $A, B$ and $C$ respectively, and $P(A) . P(B)$, and $P(C)$ be the revenue generated while they operated alone. Further, $P(A, B), P(B, C), P(C, A)$, and $P(A, B, C)$ be the coalition gain while 
they operated in e-marketplace. So, an acceptable revenue sharing solution must satisfy the following axioms (Moulin 1988):

2.2a Member rationality axiom: For a single member, the revenue allocated to it should be greater than the revenue generated while it operated alone. Otherwise, this member would not be motivated to join the coalition in e-market.

$$
\begin{aligned}
& X_{A} \geq P(A) \\
& X_{B} \geq P(B) \\
& X_{C} \geq P(C) .
\end{aligned}
$$

2.2b Coalition rationality axiom: For a coalition, the sum of the revenue allocated to its members should be at least equal to the coalition revenue generated, to encourage the participants to join the coalition in e-market.

$$
\begin{aligned}
& X_{A}+X_{B} \geq P(A, B) \\
& X_{B}+X_{C} \geq P(B, C) \\
& X_{C}+X_{A} \geq P(C, A) .
\end{aligned}
$$

2.2c Global rationality axiom: The revenue sharing scheme should satisfy the break-even requirement, which means that all the revenue generated must be completely shared among the coalition members.

$$
X_{A}+X_{B}+X_{C} \geq P(A, B, C) .
$$

If the game possesses a unique core, a unique revenue sharing is possible. However, it is hardly achieved in most of the problem. Usually, there exists a continuum of points in the core of the cooperative game. When the number of members in the coalition $(N)$ is large, the number of inequalities to be evaluated is very large. So, superadditive property of a cooperative game can be utilized to reduce the number of inequalities to be verified.

The core does not exist in some of the cases. So, it is necessary to know the conditions in which the core exists. Moulin (1988) demonstrated that if each of the coalition gain $P(S)$ is relatively small compared to grand coalition gain $P(N)$, then the core of the revenue sharing problem exists.

\subsection{Allocations based on nucleolus and shapely value}

The nucleolus and Shapely value are well-known allocation rules with a common desirable property, that they are both uniquely determined. Nucleolus (Schmeidler 1969) is the unique allocation that lexicographically minimizes the vector excesses, when the excesses are arranged in the descending order. Unlike the fractional rule, it does not satisfy any of the monotonicity criteria. Hartman \& Dror (1996) modelled allocation of benefits from holding centralized inventory in a supply chain. The authors impose three criteria that an allocation rule has to satisfy - stability of the group, justifiability (consistency between costs and benefits) and computational ease. After evaluating various allocation rules, they showed that only the nucleolus and the Shapely value satisfy both justifiability and stability criteria, but requires a large amount of computations (of order $O\left(2^{n}\right)$ ).

The centre of the core is known as nucleolus, which can be considered as a potential feasible allocation. Let $X$ be an allocation vector. Define the excess function of the coalition $S$ as 
$e(S, X)=P(S)-\sum_{i \in S} X_{i}$. Then the nucleolus of the cooperation game is the solution of the problem $\min _{X} \max _{S \subset N} e(S, X)$ (Moulin 1988). The solution of the problem implies that for each member in the coalition, it minimizes the maximum regret. The value of the excess function is a measure of the regret. It means that the larger is the revenue contribution ability of a member in the supply chain, the higher is the revenue shared from the coalition gain. Nucleolus allocation correctly reflects this rationality.

The nucleolus mechanism has certain limitations. It may not be computationally intensive depending on the number of coalitions involved. Nucleolus allocation also does not satisfy coalition monotonicity, i.e. each member of the coalition is not guaranteed to obtain increased revenue while the revenue generated by grand coalition increases. Since the incremental revenue of grand coalition is achieved by the contributions of all the members, this leads to the issue of inequity. If the core of the revenue sharing game does not exist, the nucleolus also does not exist.

The Shapely value (Shapely 1953) is an allocation rule, which satisfy both aggregate and coalitional monotonicity. It also satisfies a concept of strong monotonicity, which implies that a member's allocation should not decrease if the value of coalitions that contain it increase relative to the value of coalitions to which it does not belong. It also satisfies the following axioms (Mas-Colell et al 1995):

(i) Efficiency: This means the break-even requirement of the core, which is a fundamental requirement.

(ii) Symmetry: The Shapely value does not depend on how the supply chain members in the coalition are labelled, i.e. only their relative positions in the game as summarized by the characteristic function.

(iii) Linearity: This means that the Shapely value depends linearly on the coefficients $P(S)$ during the game.

(iv) Zero-payoff to a dummy player: If a supply chain member contributes no revenue to the game, then the Shapely value of this member is equal to zero.

The Shapely value assigned to member $i\left(X_{i}\right)$ in an $N$-member coalition is defined by (Shapely 1953)

$$
X_{i}=\sum_{S \subseteq N ; i \in S} \frac{(N-S) !(S-1) !}{N !}[P\{S\}-P\{S-\{i\}\}] \text { for } \quad i=1,2, \ldots, N
$$

The Shapely value that corresponds to member $i$ can be interpreted as the expectation of the marginal contribution/share to the total revenue allocated to this member, where the distribution of coalition arises in a particular way. To get a sense of $X_{i}$, it can be assumed that the members can collaborate or can form coalition in any arbitrary order and that all the $N$ ! orderings are equally likely. Then $X_{i}$ is the expected value, across all possible orderings, of the member $i$ 's marginal share to the total revenue generated. $[P\{S)\}-P\{S-\{i\}\}]$ is the incremental revenue shared by the member $i$ due to its joining in the coalition $S$, which is fair and desirable for each member in a cooperative game.

The Shapely value has been neglected by the researchers in the SCM fields until recently, when it was used as a solution concept for several SCM papers. Raghunathan (2003) studied the value of demand information sharing and the impact of demand correlation in a model with one manufacturer and $n$ buyers. The benefits due to information sharing are allocated 
according to the Shapely value. Granot \& Sosic (2003) studied a model of a decentralized distribution system with inventory sharing consisting of $n$ buyers, and showed that the Shapely value induces decisions that maximize the profit from information sharing, provided the SC members remain in the grand coalition. This indicates that one of the drawbacks of Shapely value approach - is that it may not belong to the core, hence some members may have an incentive to defect from the grand coalition.

Reinhardt \& Dada (2005) considered a model with $n$ firms who cooperate by pooling their critical resources. The benefits generated through this cooperation are distributed among the firms according to Shapely value. As the Shapely value may be difficult to compute with a large number of members, the authors developed an algorithm that computes it in pseudopolynomial time for a particular class of games, which they call coalition symmetric. Leng \& Parlar (2005) modelled a three level SC consisting of a supplier, a manufacturer and a buyer, who share demand information to achieve supply chain-wide cost savings. All three SC members form an information-sharing coalition, and the savings are allocated among them according to the nucleolus and Shapely value.

\section{Negotiating complex revenue sharing (RS) contract}

Before developing a scheme for revenue sharing between coalition members in e-market, it is imperative to determine a theoretical framework or policy for negotiation between contracting members in the semiconductor industry supply chain (SSC). The main motivation behind using a formal negotiation model is that it allows the SC managers to establish a framework to examine the effect of sharing profits using bargaining solutions across several structurally different supply chains.

There are two outcomes to the negotiation models as applied to supply chains. The first outcome is the mechanism of business or the contracting outcomes when SC members negotiate over the terms of the business, i.e. the contractual parameters, such as wholesale price, purchasing quantity, capacity investment, commitment to promotional activities and so on. The second outcome is the impact of the structure of the supply chain. If negotiations are considered between various members in a supply chain, it is necessary to examine how these members' relative negotiation power affects the structure of the supply chain. The approaches are the formation of bargaining alliances and the emergence of e-market intermediaries who sell insurance and commit to protect against risky negotiations.

Risk neutral members adopt the roles of suppliers and buyers across supply chain featuring both vertical and horizontal competition. In most of the cases, the supply chain attempt to coordinate the channel using a variety of contracts and the resulting profit is then shared among the members. Transfer prices, revenue sharing, buy-back pricing or quantity discounts are some of the popular mechanisms that are used to achieve this goal.

The question of how the shares are determined is not adequately addressed in the literature. In the case of risk-averse members, even the issue of coordination is usually unclear and thus the shares of profits are not adequately addressed in the literature.

An attempt has been made for prescriptive insights into the multilateral negotiations about the complex RS contracts with multiple interdependent issues. These issues should be addressed concurrently, exploiting win-win opportunities for integrative negotiations.

\subsection{Negotiation protocol}

It is assumed that there are $N$ supply chain members/echelons or partners $P_{1}, P_{2}, P_{3}, \ldots$ and $P_{N}$, who want to reach an agreement about the complex RS contract that determines 
collaboration in SSC. The set of possible contracts is defined by a formal contract space $C=\{C$ such that $C$ is a possible contract $\}$, over which the members have already gained agreement. The contract space is usually defined in an implicit way and does not allow for an exhaustive search. The members assess contracts according to their preferences by utility functions $P_{1}: C \rightarrow R, P_{2}: C \rightarrow R \ldots$ and $P_{N}: C \rightarrow R$ respectively, which represent private information. Each member aims for maximizing its utility.

The following parameters may also be considered while designing a negotiation mechanism:

- The common objective of all the members: $Z$.

- The capacity of the members: $C_{1}, C_{2}, C_{3}, \ldots$ and $C_{N}$.

- The incentive rule: $I$.

- The collaborative strategy of each member: $S_{1}, S_{2}, S_{3}, \ldots$ and $S_{N}$.

- The effect of collaboration: $U$.

- The risk of cooperation: $R$.

- The collaboration model: $Z=\left[\left(P_{1}, C_{1}, S_{1}\right),\left(P_{2}, C_{2}, S_{2}\right), \ldots,\left(P_{N}, C_{N}, S_{N}\right)\right]$.

- The outcome: $\int(U, R)$.

Taking into account asymmetric information and opportunistic behaviour of the members, the e-market intermediary (EMI) supports the negotiation process. The actions of EMI are transparent. Besides the contract space, the EMI has neither any specific knowledge about utility functions nor any special trust relationships with the SC members. The EMI repeatedly generates new probable contracts, which are accepted or rejected by the members according to specific strategies. For this, the members do not need an explicit representation of the contract space and utility functions, but are only required to be capable of assessing potential contracts compared with each other.

The EMI accepts a proposed contract (when the contract $C$ is accepted all the members after a particular round) if and only if all the members have signalled acceptance. After some termination criterion is met (with respect to a maximum number of rounds, or a maximum computation time, or maximum revenue shared), the contract eventually accepted constitutes the negotiation outcome. This negotiation protocol is rather simple, yet its effectiveness depends on two major aspects: (a) the generation of proposed contracts by EMI, and (b) the acceptance criteria of the SC members. The negotiation procedure, which may be regarded as a search process, rests on simple yes/no responses by the members having private information about specific criteria.

A simple option is some random generation of RS contracts by the EMI. Presuming that the negotiation process successively produces contracts of higher quality, a better option may be to introduce small changes to the existing contract. Such a progressive modification corresponds to the iterative selection of random neighbour solutions. The EMI may observe the negotiation process trying to infer certain regularities (i.e. of the behaviour that the members have mostly rejected the suggested contracts), which may enable for focusing the generation procedure accordingly. The proposed negotiation mechanism is depicted in figure 1 and the basic framework for the e-market supported negotiation mechanism is depicted in figure 2 .

The natural behaviour of a SC member is to accept a new contract, if and only if it is not worse than the current contract. However, the mutual application of this approach by all the members may result in a negotiation process that gets stuck soon. This risk is common in the context of local search methods, which may get stuck in the local optima of inferior quality. One approach to overcome such local optima is to adopt the classical acceptance criterion of game theory, which may be applied as the acceptance criterion of a SC member, leading 


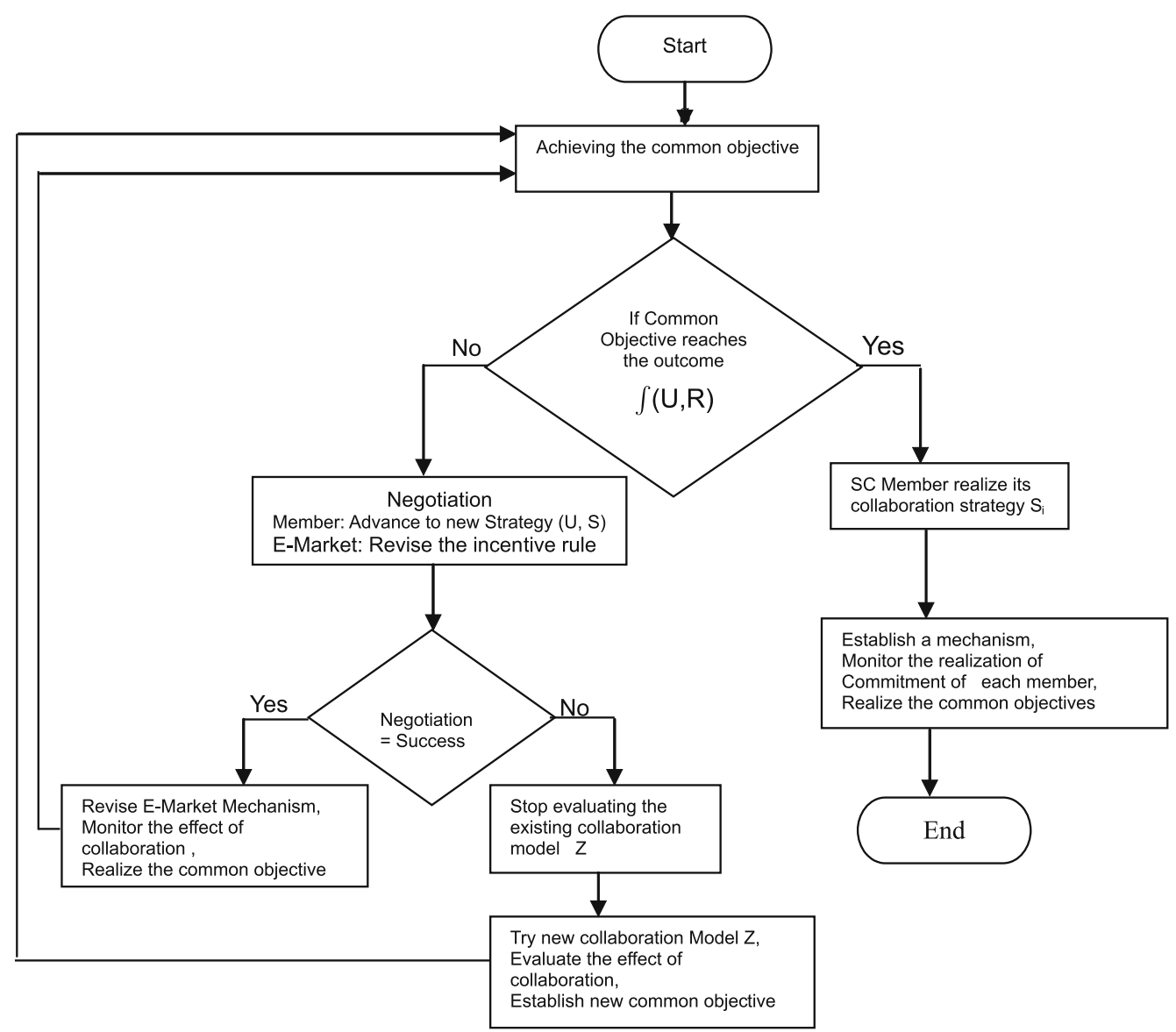

Figure 1. The proposed negotiation mechanism in E-market.

to a cooperative acceptance criterion. The crucial problem with regard to the application of cooperative acceptance criterion is about sensibly determining and adapting the Shapely value. An appropriate mechanism should be generally effective concerning a high quality negotiation outcome. Some kind of fairness must be provided. Ideally, no SC members should be able to gain profit by untruthful disclosures or corresponding distortions of its Shapely value. To facilitate a fair mechanism, the proposed method introduces two functions $X_{R j i}$ and $X_{D j i}$ (ratio and difference of the revenue allocated to the $j^{\text {th }}$ member and the $i^{\text {th }}$ member), that must be fulfilled by all the SC members.

The assessment of negotiation process depends on a basic desirable property, called Pareto optimality or efficiency of the final contract. This means that there is no other contract that improves the profit or gain of one member without making other member(s) worse off. But, there are usually a large number of Pareto optimal contracts. Under the assumption that an interpersonal comparison of gains or profit values is possible, maximization of social welfare (profit potential) may constitute a meaningful criterion for the selection among Pareto optimal contracts. However, maximization of only social welfare with some perception of fairness may not be attractive. For instance, an optimal contract might unfairly allocate the joint 


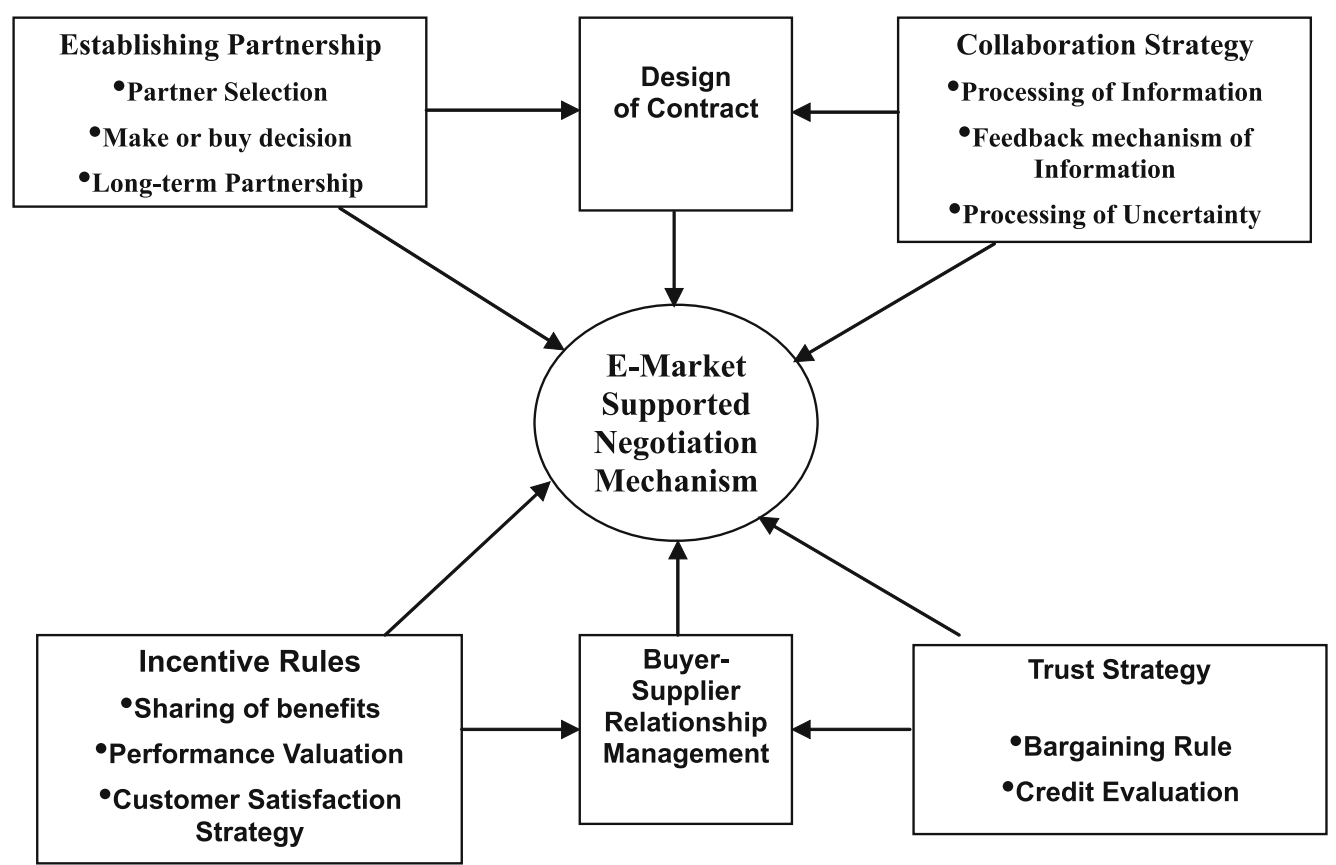

Figure 2. Framework for E-market supported negotiation mechanism.

gain due to the collaboration completely to one SC member. From a one-sided perspective, contracts may be assumed on the basis of the derivation from a hypothetical optimal contract for one member. Further, criteria are characterized by incentive-compatibility, computational traceability and low requirements for information revelation.

\subsection{Game theoretic analysis}

Under the assumption of common knowledge about the ordinal relations in the profit table, the application of the dominance criterion from game theory result in two or more SC members behaving greedily and thus obtaining the worst outcome. If one member considers every choice of the other members in turn, and selects the best answers for each choice in order to maximize its own profit function, it leads to a greedy acceptance criterion as the dominant individual strategy in every case. So, the combination of two greedy SC members represents an unambiguous Nash equilibrium, which leads to a socially dominated outcome. This situation resembles a classic prisoner's dilemma (Axelrod 1984). So, the problem arises how to overcome this strategic decision situation. As the negotiation process is an iterative game, with each round representing a separate game, the prisoner's dilemma may be overcome in iterative games (Axelrod 1984).

The proposed approach to overcome the prisoner's dilemma is as follows: The EMI basically defines mandatory acceptance rules for the members during the negotiation process. The procedure given in the previous section may be applied without change. Thus, all the SC members are enabled to determine suitable Shapely values to attain acceptance throughout the negotiation process. The EMI can easily verify that all the SC members conform to the requirements of cooperative behaviour, which is quite fair, and eventually leads to a number 
of cooperative SC members. The conscientious application of the cooperative acceptance criterion may represent incentive compatible behaviour concerning the honest revelation of private information. Otherwise, a member would have to partly accept worse contract. The formal proof of such an approach is beyond the scope of the current research.

In summary, the contributions of this suggested approach are: (a) design of a general multilateral negotiation procedure concerning fair cooperative acceptance criterion, and (b) the negotiation mechanism is simple and general, i.e. no exploitation of domain specific knowledge. The design of the cooperative acceptance criterion allows the definition of the negotiation rules that can be verified to enable the SC members to collaborate in a cooperative manner.

\section{Economic value model (Revenue generation model)}

The economic value (revenue generation capability) of a coalition in an e-marketplace is typically dependent on three factors (Subramanian \& Shaw 2004): (1) price benefits, (2) transaction cost benefits, and (3) technology lock-in costs. Mathematically, it is represented as

$$
\begin{aligned}
\text { Economic value }= & \text { Price benefits }+ \text { Transaction cost benefits } \\
& - \text { Technology lock-in costs }
\end{aligned}
$$

price benefits $(\mathrm{PB})$ result from the potential price reduction off the market price, which can be estimated for product/component category as lowest negotiated contract price and the average market price.

$$
\mathrm{PB}=\text { Lowest negotiated contract price }- \text { Average market price. }
$$

Transaction cost benefits (TCB) result from savings in search cost, negotiation and contracting cost, and coordination cost. Search cost is concerned with locating an appropriate supplier/contract manufacturer for a product/component category. Negotiation and contracting costs are concerned with the process of bidding, evaluating and selecting supplier/contract manufacturer, and contract writing. Coordination costs involve processing and following-up orders.

$$
\begin{gathered}
\mathrm{TCB}=\text { Savings in }(\text { Search cost }+ \text { Negotiation and contracting cost } \\
+ \text { coordination cost }) .
\end{gathered}
$$

Technology lock-in cost (TLC) results from choosing and using specific B2B system to participate/transact in e-market, which include switching cost and costs due to opportunistic behaviour by contracted suppliers. These costs are offset by the extent of protection from uncertainty.

$$
\text { TLC }=\text { Switching cost }+ \text { Opportunity cost }+ \text { System cost } .
$$

So, the total revenue (TR) can be the contribution of all these cost.

$$
\begin{aligned}
\mathrm{TR} & =\mathrm{PB}+\mathrm{TCB}-\mathrm{TLC} \\
& =F+M(i)(\text { Say }) .
\end{aligned}
$$


$F$ in the above expression is the fixed cost associated with revenue generation, which equally serve all the members in the supply chain coalition. The fixed cost function may be discontinuous. However, $F$ is the minimum amount of revenue contributed by the supply chain members due to cooperation/coordination/collaboration. $M(i)$ represents the marginal cost that corresponds to the amount of revenue contributed to TR by a member to the coalition depending on his ability in e-market. In the present analysis, the supply chain members and/or echelons are assumed to be used interchangeably.

\section{Analysis of RS problem through Shapely value approach}

Shapely value is a concept of cooperative game theory which assigns each coalition game a unique solution based on the axiomatic foundations (Myerson 1991). In a supply chain coalition of $N$-members/echelons in e-market, the total revenue allocated to the members is given by

$$
P(N)=F+\sum_{i=1}^{N} M(i) .
$$

It is assumed that

$$
\begin{aligned}
P\{\phi\} & =0 . \\
P(S) & =\operatorname{Max}_{i \in S}[T R\{i\}] \\
P(K) & =F+\sum_{i=1}^{K} M(i),
\end{aligned}
$$

where $S$ is any possible coalition of members in semiconductor industry supply chain and $i(i=1,2, \ldots, N)$ denotes a specific member or echelon in the coalition. Equation (7) implies that the total revenue allocated/generated due to the coalition of the members in the supply chain equals the combination of fixed cost and marginal cost. Equation (9) ensures that if there is no member in the coalition, the total revenue generated is zero. Equations (9) and (10) depict that the total economic value (revenue) for any sub-set (sub-coalition) of members of the supply chain is equal to the total revenue generated due to the presence of the last member within that sub-set.

In order to transform Equation (6) representing the Shapely value, the present method uses the solution of Littlechild \& Owen (1973). The revenue structure includes a fixed cost element, which is not present in the above model. As the fixed cost in the proposed framework is used for all factors in the economic value that equally serve all the members in the coalition, the Shapely value in Equation (5) can be transformed as

$$
\begin{aligned}
& X_{i}=\sum_{Y=1}^{i} \frac{T R(Y)-T R(Y-1)}{N-Y+1} \text { for } i=1,2, \ldots, N \\
& X_{1}=\frac{T R\{1\}}{N}=\frac{F+M(1)}{N} \\
& X_{2}=\frac{T R\{1\}-T R\{0\}}{N}+\frac{T R\{2\}-T R\{1\}}{N-1}=X_{1}+\frac{M(2)}{N-1} \text { and }
\end{aligned}
$$




$$
X_{3}=\frac{T R\{1\}-T R\{0\}}{N}+\frac{T R\{2\}-T R\{1\}}{N-1}+\frac{T R\{3\}-T R\{2\}}{N-2}=X_{2}+\frac{M(3)}{N-2}
$$

So, it can be generalized by the expression

$$
X_{i}=X_{i-1}+\frac{M(i)}{N-Y+1} .
$$

Suppose $i$ and $j$ be any two arbitrary members in the $N$-member coalition, where $j>i$. Define the ratio between the revenue allocated to $j^{\text {th }}$ the member and $i^{\text {th }}$ member (according to Shapely solution) by $X_{R j i}$, and the difference between the revenue allocated to the $j^{\text {th }}$ member and that allocated to $i^{\text {th }}$ member by $X_{D j i}$.

So,

$$
X_{R j i}=\frac{X_{j}}{X_{i}}
$$

and

$$
X_{D j i}=X_{j}-X_{i}
$$

An alternative to distribute the revenue is to identify the contribution of each member to the grand coalition. A complication that arises in estimating this revenue generation capability is the number of alternative combinations of the SC members (or coalitions in the context of cooperative game theory) that needs to be considered. Based on the mathematical expression (7), the revenue of all possible coalitions can be computed. Let $L$ be a possible coalition and $P(L)$ be the associated revenue gain due to cooperation. Then a transformation can be made, which is

$$
P(L)=\sum_{i \in L} C(i)-C(L),
$$

where $C$ (.) is the contribution to the revenue. Using this transformation, the cost allocation problem can be replaced with a revenue sharing problem (Moulin 1988).

\subsection{Tests for superadditive property of a revenue sharing (RS) game}

In order to prove that the coalition structure is stable, it is essential to test whether the RS game is superadditive or not. Let $S$ and $T$ be two sub-coalitions in the supply chain without intersection. Suppose $R_{S}$ is the revenue generated when $S$ operates on its own, with a coalition gain $P(S)$.

$$
P(S)=\sum_{i \in S} C(i)-C(S)
$$

Suppose $R_{T}$ is the revenue generated when $T$ operates on its own, with a coalition gain $P(T)$.

$$
P(T)=\sum_{i \in T} C(i)-C(T) .
$$

If no congestion exists anywhere in the supply chain network operating in e-market, all the members in the coalition are assumed to be identical. So, a maximum of $R_{S}+R_{T}$ amount of revenue would be generated when the sub-coalition $S \cup T$ operates. This means that

$$
C(S \cup T) \leq C(S)+C(T) .
$$


Further,

$$
\sum_{i \in S \cup T} C(i)=\sum_{i \in S} C(i)=\sum_{i \in T} C(i)
$$

So,

$$
\begin{aligned}
P(S)+P(T) & =\sum_{i \in S} C(i)-C(S)+\sum_{i \in T} C(i)-C(T) \\
& =\sum_{i \in S \cup T} C(i)-[C(S)+C(T)] \\
& \leq \sum_{i \in S \cup T} C(i)-C(S \cup T) \\
& \leq P(S \cup T)
\end{aligned}
$$

So,

$$
P(S)+P(T) \leq P(S \cup T) .
$$

This implies that if all the members in the supply chain coalition are identical due to their participation in the e-market, and the supply chain system is unconstrained, then the revenue sharing game is superadditive. So, the coalition structure is stable, i.e., it is possible to obtain a contractually stable network. This property of the RS game reduces the number of inequalities to be verified for the existence of a core.

\subsection{Tests for convexity of a revenue sharing (RS) game}

If an allocation does not lie in the core, it is not stable and hence the mechanism is not attractive to the members of the coalition. So, the conditions under which the Shapely value allocation lies in the core must be addressed. Literature suggests that if a cooperative game is convex, then the core of the game is non-empty and the Shapely value allocation lies in the core (Moulin 1988). A game is convex, if

$$
P(S)+P(T) \leq P(S \cup T)+P(S \cap T) \text { if } S, T \subset N .
$$

Suppose $S$ and $T$ be two supply chain coalitions operating in e-market where no congestion occurs. Assume all the members similar. Let $R_{S}$ and $R_{T}$ be the revenue generation capabilities of $S$ and $T$ respectively, which are equal to the total revenue generation capabilities of the coalitions $S$ and $T$ substracting the capabilities of the system members on their own. Also assume $S \subset T$ and $R_{S} \leq R_{T}$.

The contribution to the revenue by member $i$ when it joins the coalition $T$ is less than when it joins the coalition $S$. So, the following inequality holds:

$$
C(T \cup\{i\})-C(T) \leq C(S \cup\{i\})-C(S) .
$$


Based on the definition of the benefit function, the above equation can be represented as

$$
\begin{aligned}
& {\left[\sum_{j \in T \cup\{i\}} C(j)-P(T \cup\{i\})\right]-\left[\sum_{j \in T} C(j)-P(T)\right]} \\
& \leq\left[\sum_{j \in S \cup\{i\}} C(j)-P(S \cup\{i\})\right]-\left[\sum_{j \in T} C(j)-P(S)\right] .
\end{aligned}
$$

Further,

$$
\begin{aligned}
& \sum_{j \in T \cup\{i\}} C(j)-\sum_{j \in T} C(j)=C(i) \text { and } \\
& \sum_{j \in S \cup\{i\}} C(j)-\sum_{j \in S} C(j)=C(i)
\end{aligned}
$$

So,

$$
-P(T \cup\{i\})+P(T)+C(i) \leq-P(S \cup\{i\})+P(S)+C(i),
$$

which implies that

$$
P(S \cup\{i\})-P(S) \leq P(T \cup\{i\})-P(T) .
$$

This implies that under the assumptions stated above, the revenue sharing game is a convex game, i.e. if a supply chain member joins a larger coalition, more revenue would be generated for this coalition than while it joins a smaller coalition. The literature suggests that a convex game is always a balanced game (Moulin (1988)).

\subsection{Shapely value for any member in SC coalition}

The revenue generated by the first member is $F+M(1)$. As the revenue generated by the two-member coalition relies on the marginal contribution of the second member, the revenue generated is $F+M(1)+M(2)$. In general, the revenue generated by a supply chain coalition with $j$ members is $F+M(1)+M(2)+\cdots+M(j)$. So, the fixed cost $(F)$ and $M(1)$ are equally shared/divided among all the members of the coalition, $M(2)$ is shared/divided among all the members from second member and beyond, and $M(j)$ is divided among all the members from the $j^{\text {th }}$ member and beyond.

Example: Suppose $N=4$. Then according to the Shapely scheme, the revenue shared by the first member is

$$
X_{1}=\frac{F+M(1)}{4} .
$$

The revenue shared by the second member is

$$
X_{2}=\frac{F+M(1)}{4}+\frac{M(2)}{3} .
$$

The revenue shared by the third member is

$$
X_{3}=\frac{F+M(1)}{4}+\frac{M(2)}{3}+\frac{M(3)}{2} .
$$


The revenue shared by the fourth member is

$$
X_{4}=\frac{F+M(1)}{4}+\frac{M(2)}{3}+\frac{M(3)}{2}+M(4) .
$$

So, the sum of the revenue shared by the members is

$$
X_{1}+X_{2}+X_{3}+X_{4}=P\{4\}
$$

An alternative approach for allocating this revenue among the coalition members is to average member $\mathrm{j}$ 's marginal contribution to the total revenue generated across all possible coalition combinations. For $N=4$, the revenue sharing by individual members is depicted in table 1. Similar estimates can be made as the number of coalition members increases. It has been observed that the allocated revenue increases with the number of members in the coalition. Further, it can be inferred that as the share of fixed cost increases in the total cost, the revenue shared by all the members will be equal.

In general, the Shapely value for any member $j$ in the $N$-member coalition

$$
X_{j}=\frac{F}{N}+\frac{M(1)}{N}+\frac{M(2)}{N-1}+\cdots+\frac{M(j-1)}{N-j+2}+\frac{M(j)}{N-j+1} .
$$

This can be simplified as

$$
X_{j}=\frac{F}{N}+\sum_{Y=N-j+1}^{N} \frac{M(N-Y+1)}{Y} .
$$

Further, the ratio between the revenue allocated to the $j^{\text {th }}$ member and that allocated to $i^{\text {th }}$ member is given by

$$
X_{R j i}=\frac{\frac{F}{N}+\sum_{Y=N-j+1}^{N} \frac{M(N-Y+1)}{Y}}{\frac{F}{N}+\sum_{Y=N-i+1}^{N} \frac{M(N-Y+1)}{Y}} .
$$

The difference between the revenue allocated to the $j^{\text {th }}$ member and that allocated to the $i^{\text {th }}$ member is given by

$$
X_{D j i}=\sum_{Y=N-j+1}^{N} \frac{M(N-Y+1)}{Y}-\sum_{Y=N-i+1}^{N} \frac{M(N-Y+1)}{Y} .
$$

It is clear from Equation (24) and (25) that $X_{R j i}$ increase with $j$ and $X_{D j i}$ decrease with $j$, when $M(i) \neq 0$ for $i=1,2, \ldots, N$. The above analysis leads to the following observations:

According to Shapely scheme of revenue sharing, the total revenue of $N$-member coalition in the supply chain is simply allocated to each member based on its relative position in the coalition.

The ratio and difference function $\left(X_{R j i}\right.$ and $\left.X_{D j i}\right)$ will provide a practical tool for comparing the revenue shared by each member in the coalition. 


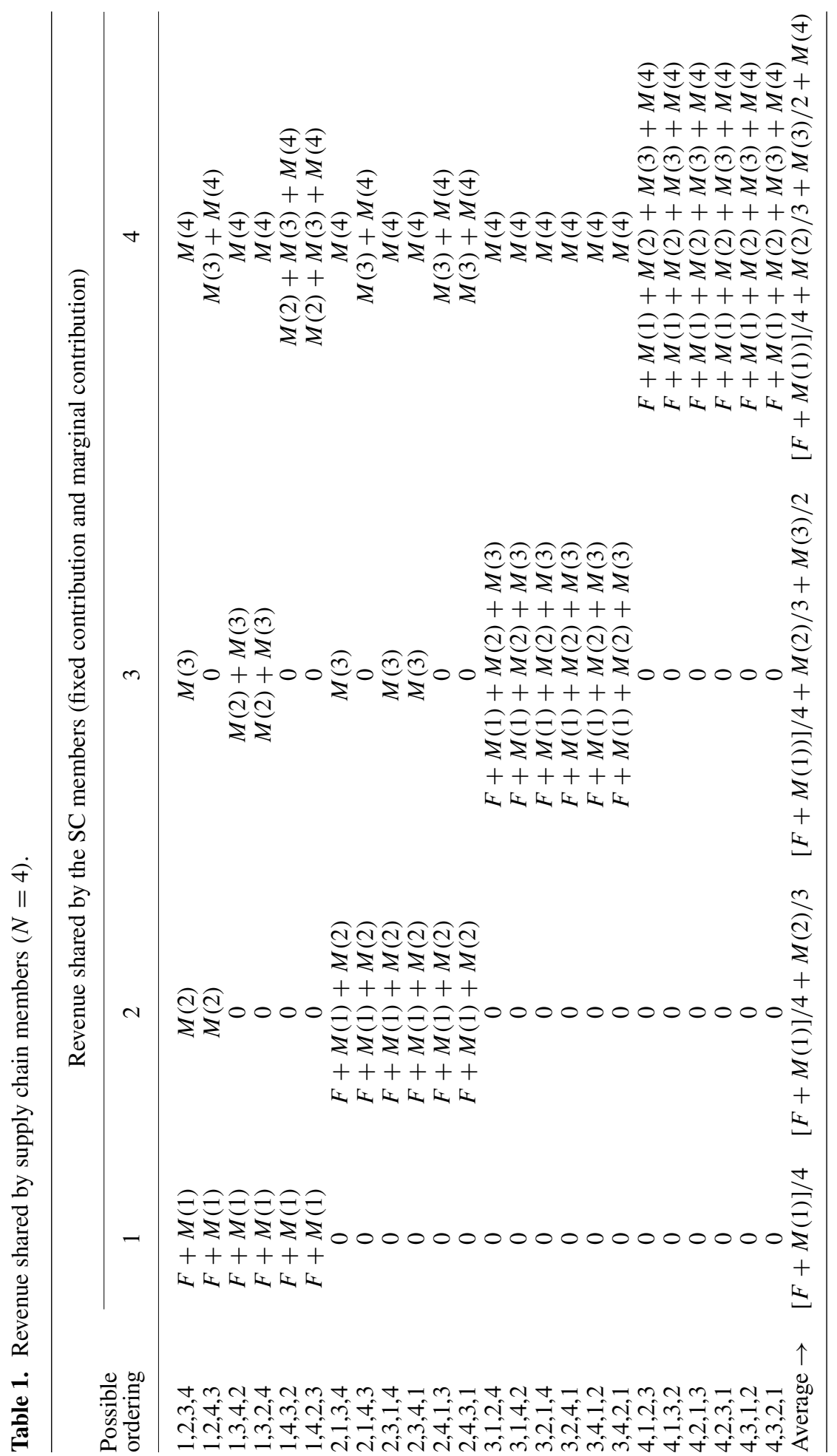


5.3a Shapely value analysis for same marginal contribution by coalition members: Assume that the marginal contributions of the members in the supply chain coalition are the same, i.e. $M(i)=M(j)=M$ for all $i, j=1,2, \ldots, N$. Then Equation (23) can be expressed as

$$
X_{j}=\frac{F}{N}+M \sum_{Y=N-j+1}^{N} \frac{1}{Y}
$$

Let

$$
S(Z)=\sum_{Y=1}^{y} \frac{1}{Y}
$$

But,

$$
\begin{aligned}
& \sum_{Y=1}^{y} \frac{1}{Y} \approx \ln (y) \\
& X_{j}=\frac{F}{N}+M[S(N)-S(N-j)]
\end{aligned}
$$

This gives rise to

$$
X_{j}=\frac{F}{N}+\ln \left(\frac{N}{N-j}\right)^{M} .
$$

Further, under the assumption of same marginal contribution by each supply chain member, we have

$$
X_{D j i}=M\left[\sum_{Y=N-j+1}^{N} \frac{1}{Y}-\sum_{Y=N-i+1}^{N} \frac{1}{Y}\right] .
$$

If $j$ is the next member in the supply chain after $i$ (put $j+1=j$ and $j=i$ ), we have

$$
\begin{aligned}
X_{D j i} & =M\left[\sum_{Y=N-j}^{N} \frac{1}{Y}-\sum_{Y=N-j+1}^{N} \frac{1}{Y}\right] \\
X_{D j i} & =M\left[\frac{1}{N-j}\right] .
\end{aligned}
$$

So, $X_{D j i}$ monotonically increases with $j$.

Further, Equation (24) can be rewritten as

$$
X_{R j i}=\frac{\frac{F}{N}+M \sum_{Y=N-j+1}^{N} \frac{1}{Y}}{\frac{F}{N}+M \sum_{Y=N-i+1}^{N} \frac{1}{Y}}=\frac{\frac{F}{N \cdot M}+\sum_{Y=N-j+1}^{N} \frac{1}{Y}}{\frac{F}{N \cdot M}+\sum_{Y=N-i+1}^{N} \frac{1}{Y}} .
$$

So, the ratio between the revenue allocated to the $(j+1)^{\text {th }}$ member and that allocated to $j^{\text {th }}$ member increase with $j$, only when

$$
\frac{\frac{F}{N \cdot M}+\sum_{Y=N-j+1}^{N} \frac{1}{Y}}{\frac{F}{N \cdot M}+\sum_{Y=N-j+2}^{N} \frac{1}{Y}} \prec \frac{\frac{F}{N \cdot M}+\sum_{Y=N-j}^{N} \frac{1}{Y}}{\frac{F}{N \cdot M}+\sum_{Y=N-j+1}^{N} \frac{1}{Y}} .
$$


Further simplification leads to

$$
\sum_{Y=N-j+1}^{N} \frac{1}{Y} \succ 1-\frac{F}{N \cdot M} .
$$

Equation (31) represents the condition for which the ratio $X_{R j i}$ increases with $j$. The condition for which the ratio $X_{R j i}$ decreases with $j$ is $\sum_{Y=N-j+1}^{N} \frac{1}{Y} \prec 1-\frac{F}{N \cdot M}$.

By analysing the expressions (27) and (31), it is clear that $X_{R j i} \succ 1$ for all $i=1,2, \ldots, N-$ 1 and $j=2,3, \ldots, N$ and $j \succ i$. If the share of $F$ increases, $\frac{F}{N \cdot M}$ increases, which results in a decrease of $X_{R j i}$. Further, $X_{D j i}$ is independent of the fixed cost component. So, it is inferred that $X_{R j i}$ decreases with the increase in the share of fixed revenue in the total revenue, whereas $X_{D j i}$ is independent of the fixed revenue. The above analysis leads to the following observations:

- Equation (27) provides a quick and simple method for estimating the revenue shared by any member in the coalition according to the Shapely scheme.

- Further, the difference $[S(N)-S(N-j)]$ increases with $j$ at a slow pace. This implies that while the revenue shared by each member increases down the chain towards the buyer, the difference in revenue shared between the members is relatively less.

- Though the Shapely scheme allocates more revenue to the member down the supply chain in coalition, the percentage change between the revenues allocated to successive members is not monotonic. In other words, the ratio between the revenues allocated to two successive members first decreases and then increases as one move down the coalition structure of the supply chain.

- As the level of the fixed cost component within the total revenue increases, the discrepancies in the revenue shared by the members in the coalition decreases. This happens only when there is a strong relationship among the members in the supply chain.

- The percentage change in the revenue allocated to any member relative to the previous member is dependent upon the share of the fixed revenue, which decreases with the level of fixed cost.

$5.3 b$ Shapely value analysis when the number of coalition members $(N)$ is large: Again by assuming that the marginal contributions of the members in the supply chain coalition are the same, i.e. $M(i)=M(j)=M$ for all $i, j=1,2, \ldots, N$, we have

$$
X_{D j i}=M\left[\sum_{Y=N-j+1}^{N} \frac{1}{Y}-\sum_{Y=N-i+1}^{N} \frac{1}{Y}\right] .
$$

For large values of $N$, we have

$$
X_{D j i}=M \sum_{Y=N-j+1}^{N-i} \frac{1}{Y} .
$$

So, it is obvious that for very large value of $N$, Equation (31) converges to zero.

$$
X_{R j i}=\frac{\frac{F}{N \cdot M}+\sum_{Y=N-j+1}^{N} \frac{1}{Y}}{\frac{F}{N \cdot M}+\sum_{Y=N-i+1}^{N} \frac{1}{Y}} .
$$


The maximum value of $X_{R j i}$ is $\frac{\frac{F}{N \cdot M}+\frac{j}{N-j+1}}{\frac{F}{N \cdot M}+\frac{i}{N}}$, and the minimum value of $X_{R j i}$ is $\frac{\frac{F}{N \cdot M}+\frac{j}{N}}{\frac{F}{N \cdot M}+\frac{i}{N-i+1}}$.

So, we have

$$
\frac{\frac{F}{N \cdot M}+\frac{j}{N}}{\frac{F}{N \cdot M}+\frac{i}{N-i+1}} \leq X_{R j i} \leq \frac{\frac{F}{N \cdot M}+\frac{j}{N-j+1}}{\frac{F}{N \cdot M}+\frac{i}{N}} .
$$

Further simplification implies that

$$
\frac{\frac{(F / M)+j}{N}}{\frac{(F / M)+i}{N-i+1}} \leq X_{R j i} \leq \frac{\frac{(F / M)+j}{N-j+1}}{\frac{(F / M)+i}{N}}
$$

For very large values of $N$, the value of $X_{R j i}$ represented in Equation (24) converge to $\frac{(F / M)+j}{(F / M)+i}$. So, it has been observed from the above analysis that when the number of members in the coalition is very large and the marginal contribution of each member to the total revenue is same, all the members share almost equal revenue.

5.3c Shapely value analysis when fixed cost varies with $N$ : By assuming a large value of $N$, we have $X_{D j i}=M \sum_{Y=N-j+1}^{N-i} \frac{1}{Y}$. It is clear from this expression that $X_{D j i}$ decreases with the number of members in the coalition.

Further, we have

$$
X_{j}=\frac{F}{N}+M[S(N)-S(N-j)] .
$$

The difference $[S(N)-S(N-j)]$ decreases with the value of $N$. So, the revenue allocated to any $j^{\text {th }}$ member drops with the number of members in the coalition. Further, we have

$$
X_{R j i}=\frac{\frac{F}{N \cdot M}+\sum_{Y=N-j+1}^{N} \frac{1}{Y}}{\frac{F}{N \cdot M}+\sum_{Y=N-i+1}^{N} \frac{1}{Y}} .
$$

If it is assumed that $x_{R j i}(j \succ i, i=1,2, \ldots, N-1$ and $j=2,3, \ldots, N)$ decreases with the number of members in the coalition, we have

$$
\frac{\frac{F}{N \cdot M}+\sum_{Y=N-j+1}^{N} \frac{1}{Y}}{\frac{F}{N \cdot M}+\sum_{Y=N-i+1}^{N} \frac{1}{Y}} \succ \frac{\frac{F}{N \cdot M}+\sum_{Y=N-j+2}^{N+1} \frac{1}{Y}}{\frac{F}{N \cdot M}+\sum_{Y=N-i+2}^{N+1} \frac{1}{Y}} .
$$

The RHS of Equation (33) can be represented by

$$
\frac{\frac{F}{N \cdot M}+\sum_{Y=N-j+2}^{N+1} \frac{1}{Y}}{\frac{F}{N \cdot M}+\sum_{Y=N-i+2}^{N+1} \frac{1}{Y}}=\frac{\frac{F}{N \cdot M}+\sum_{Y=N-j+1}^{N} \frac{1}{Y}+\left(\frac{1}{N+1}-\frac{1}{N-j+1}\right)}{\frac{F}{N \cdot M}+\sum_{Y=N-i+1}^{N} \frac{1}{Y}+\left(\frac{1}{N+1}-\frac{1}{N-i+1}\right)} .
$$

So, we have

$$
\frac{j(N+1)(N-i+1)}{i(N+1)(N-j+1)} \succ \frac{\frac{F}{N \cdot M}+\sum_{Y=N-j+1}^{N} \frac{1}{Y}}{\frac{F}{N \cdot M}+\sum_{Y=N-i+1}^{N} \frac{1}{Y}} .
$$


Further simplification results in

$$
\frac{(j-i)(N+1)}{i(N-j+1)} \succ \frac{\sum_{Y=N-j+1}^{N-i} \frac{1}{Y}}{\frac{F}{N \cdot M}+\sum_{Y=N-i+1}^{N} \frac{1}{Y}} .
$$

The maximum value of $\frac{\sum_{Y=N-j+1}^{N-i} \frac{1}{Y}}{\frac{F}{N \cdot M}+\sum_{Y=N-i+1}^{N} \frac{1}{Y}}$ is given by

$$
\frac{\operatorname{Max} \cdot\left[\frac{1}{N-j+1}+\frac{1}{N-j+2}+\cdots+\frac{1}{N-i}\right]}{\frac{F}{N \cdot M}+\operatorname{Min} \cdot\left[\frac{1}{N-i+1}+\frac{1}{N-i+2}+\cdots+\frac{1}{N}\right]}=\frac{\left(\frac{j-i}{N-j+1}\right)}{\frac{F}{N \cdot M}+\frac{i}{N}}
$$

So, we have

$$
\frac{\sum_{Y=N-j+1}^{N-i} \frac{1}{Y}}{\frac{F}{N \cdot M}+\sum_{Y=N-i+1}^{N} \frac{1}{Y}} \leq \frac{\left(\frac{j-i}{N-j+1}\right)}{\frac{F}{N \cdot M}+\frac{i}{N}} .
$$

This implies that

$$
\begin{aligned}
\frac{\left(\frac{j-i}{N-j+1}\right)}{\left(\frac{i}{N+1}\right)} \succ \frac{\left(\frac{j-i}{N-j+1}\right)}{\frac{F}{N \cdot M}+\frac{i}{N}} & \Rightarrow \frac{F}{N \cdot M}+\frac{i}{N} \succ \frac{i}{N+1} \\
& \Rightarrow \frac{F}{N \cdot M} \succ \frac{-i}{N(N+1)} .
\end{aligned}
$$

This relationships hold as the LHS is always positive. So, our assumption that $X_{R j i}(j \succ$ $i, i=1,2, \ldots, N-1$ and $j=2,3, \ldots, N)$ decreases with the number of members in the coalition holds.

The above analysis indicates that for large number of coalition members $(N)$ with fixed cost of revenue generation depending on $N$, the revenue sharing curve flattens with the $N$, i.e. the supply chain members/echelons share approximately equal revenue.

\subsection{Simulation and analysis of results}

In the figures given in the Appendix 1, it has been demonstrated that the revenue allocated among the supply chain echelons $(3,4,5$, and 6 echelons). We have compared the revenue allocation for different shares of fixed cost due to cooperation in the e-market. It has been observed that while the revenue allocated among various echelons increase with the position of the echelon in the supply chain, the curve flattens as the share of fixed cost increases. The results are simulated and presented for a fixed cost share of $0 \%, 10 \%, 20 \%, 30 \%, 40 \%, 50 \%$, $60 \%, 70 \%, 80 \%, 90 \%$ and $100 \%$ of the total revenue generated. It has also been observed that the percentage change in the revenue shared by a particular echelon relative to the preceding echelon is dependent upon the share of the fixed cost in the total revenue. Analysis has also been made regarding average percentage change in the revenue shared by every two successive echelons as a function of the number of echelons in the supply chain. It has been observed that the average percentage change in the cost allocated to all successive echelons is reduced with the number of echelons in the supply chain. Further, as the share of the fixed cost increases, the average percentage change in the cost allocated to two successive echelons decreases for given number of echelons in the supply chain. 


\section{Concluding remarks}

The current research proposes a theoretical framework for negotiation between contracting members in the semiconductor industry supply chain (SSC) prior to the development of a revenue sharing scheme and coalition members in e-market. When a supply chain member joins an existing coalition, it is difficult to determine the revenue generating capability of the member before joining. So, it is tedious to ensure conforming to the conditions of a convex game. The Shapely value has a property of marginality, which means that the marginal contribution of a coalition member is the only factor that decides his revenue sharing capability. It also possesses desirable properties that make it suitable for an equitable and rational revenue sharing approach. Further, as compared to nucleolus approach, the Shapely scheme of revenue sharing is relatively easier to estimate, unless the number of coalitions is very high, and it is unique. The proposed method applies Shapely value approach for allocating the economic value or revenue generated among all the echelons of the SSC. According to the proposed revenue sharing mechanism in e-market, each echelon's share in the total revenue increases with the number of echelon in the SSC in a particular manner.

The current research showed the following:

(a) The conditions under which the ratio and difference of the revenue shared between any two successive echelons either rise or fall with an increase in the number of echelons.

(b) The fixed cost allocated to any given echelon drops with the number of echelons in the supply chain (when the total revenue are increased proportionally).

(c) The ratio and the difference between the revenue shared among any two echelons decrease with the number of echelons in the SC.

(d) The ratio and the difference between the revenue shared among any two echelons decrease with the share of the fixed cost in the total revenue.

The research has several economic implications:

(a) This approach aims at providing a benchmark for the fair distribution of revenue as a function of various coordination variables (fixed cost, marginal cost and the number of echelons in the SC).

(b) The approach may enable to explore the various profit centers within the SC.

Both Shapely approach and nucleolus approach gives better revenue sharing results than the proportional rule (Nagarajan \& Sosic 2006). This demonstrates the usefulness of the proposed axiomatic framework. Like any other market design, the proposed scheme in e-market does not preclude gaming by the coalition members. The motivation behind such a scheme is to align the supply chain members' cost structure with the bidding during auction and bargaining for e-procurement. Though Shapely value approach is appealing from the perspective of fairness, it may not be a reasonable prediction of the outcome of the game. This is due to the fact that the Shapely value need not be in the core and there exists some sub-set of supply chain members who can deviate and improve their performance. Further, the paper has not provided any assessment of relative impact of e-market forces in revenue sharing, which may be a scope of future research. 


\section{Appendix 1}

Shapely value analysis for varying fixed costs and marginal costs.
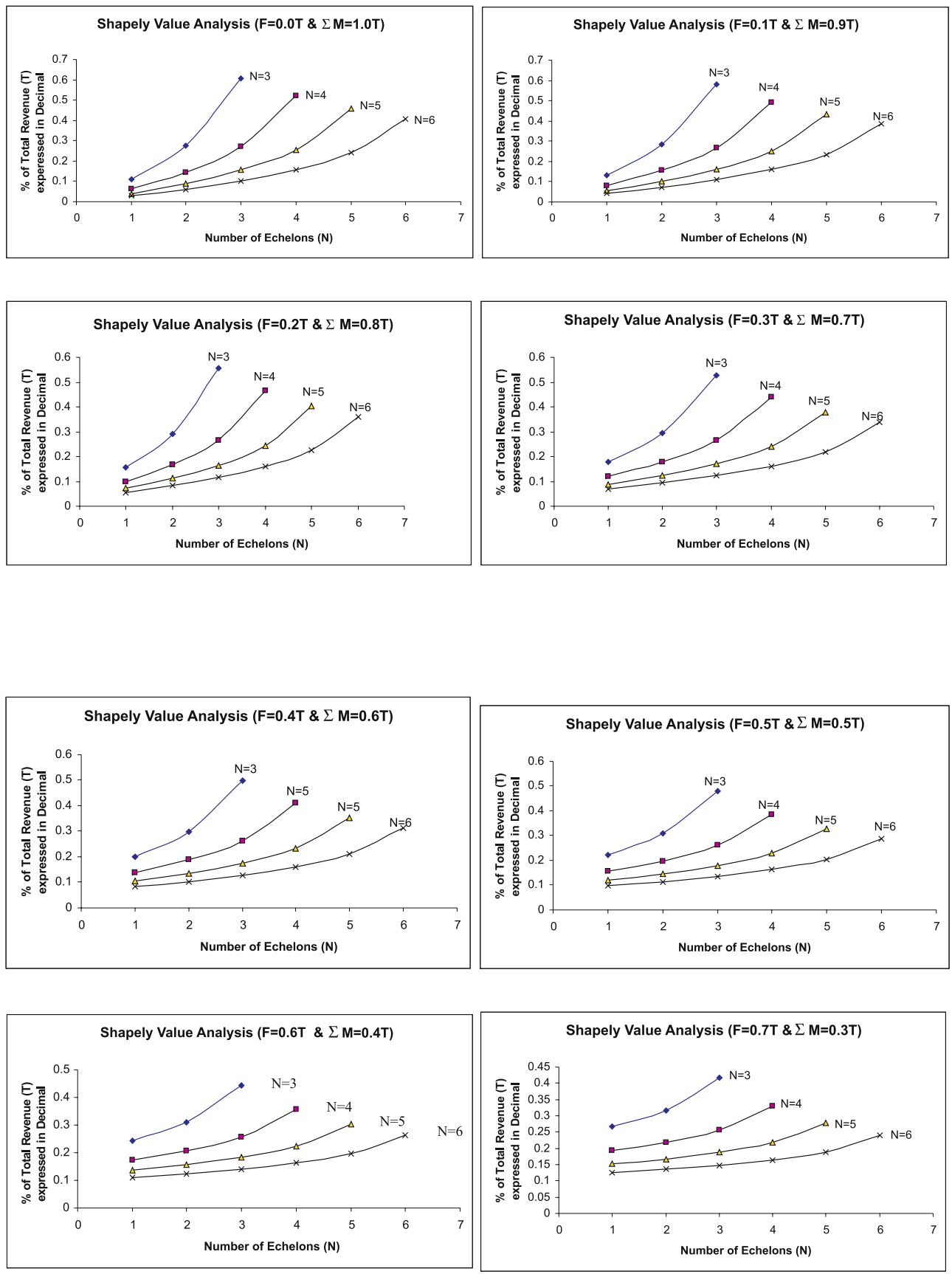

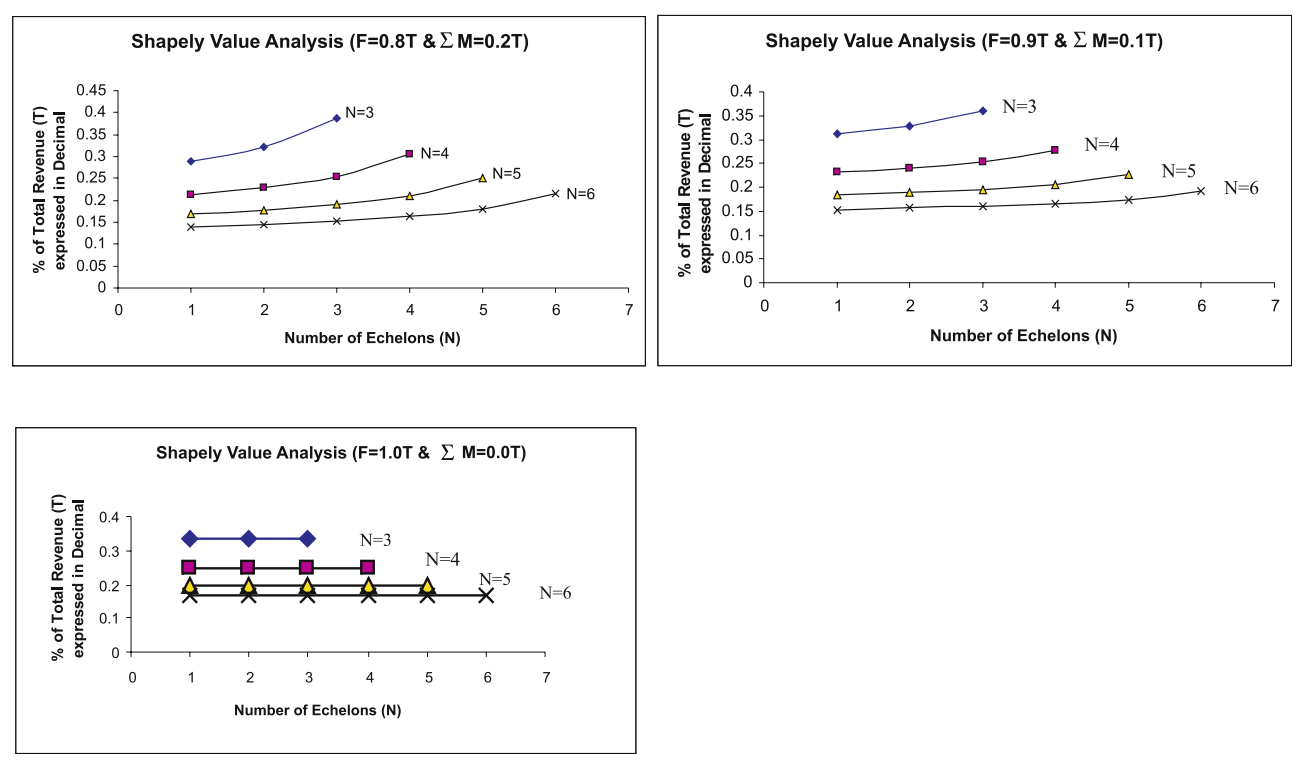

\section{References}

Aumann R J, Dreze J H 1974 Cooperative games with coalition structures. Int. J. Game Theory 3: $217-237$

Aumann R J, Myerson R B 1988 Endogenous formation of links between players and of coalitions: An application of the Shapely value, in the Shapely Value: Essays in Honor of Lloyd S Shapely (Alvin-E Roth (ed.)) Cambridge, U.K. 175-191

Axelrod R 1984 The Evolution of Cooperation, Basic Books, New York

Bela V, Goyal S 2000 A non-cooperative model of network formation. Econometrica 68: 1181-1229

Cachon G, Netessine S 2004 Game theory in supply chain analysis. In Handbook of Quantitative Supply Chain Analysis: Modelling in the eBusiness Era. (eds.) David Simchi-Levi, S David Wu, Zuo-Jun (Max) Shen Kluwer

Cachon G, Lariviere M 2005 Supply chain coordination with revenue sharing contracts. Management Science 51(1): 30-44

Cachon G P, Netessine S 2004 Game theory in supply chain analysis, in D Simchi-Levi, S D Wu, Z-J Shen (eds.) Handbook of Quantitative Supply Chain Analysis: Modelling in E-business Era, Boston, MA, Kluwer Academic Publisher, 13-66

Caulier J-F, Mauleon A, Vannetelbosch V 2007 Contractually stable networks, Working paper, University of Louvain, Belgium, 1-10

Chopra S, Meindl P 2004 Supply Chain Management: Strategy, Planning and Operation, Second Edition, (NJ: Prentice Hall, Englewood Cliffs)

Eisenhardt K M 1989 Agency theory: An assessment and review. Academy of Management Review 14(1): 57-74

Feinberg Y 1998 An incomplete cooperation structure for a voting game can be strategically stable. Games and Economic Behaviour 24: 2-9

Fisher M 1997 What is the Right Supply Chain for your Product? Harvard Business Review, MarchApril 105-116

Fundenberg D, Tirole J 2005 Game theory, first Indian reprint, (New Delhi: Ane Books)

Gillies D B 1959 Solutions to general non-zero sum games, In: A W Tucker, R D Luce (eds.) Contributions to the Theory of Games, Vol. IV, Princeton University Press, Princeton, NJ, 47-83 
Goyal S, Joshi S 2003 Networks of collaboration in oligopoly. Games and Economic Behaviour 43(1): $57-85$

Goyal S, Joshi S 2006 Bilateralism and free trade. Int. Economic Rev. 47(3): 749-778

Granot D, Sosic G 2003 A three stage model for a decentralized distribution system of retailers. Operations Res. 51: 771-784

Greenberg J 1994 Coalition structures, in Handbook of Game Theory, R J Aumann, S Hart (eds.) Elsevier Science, B.V. North Holland 2: 1305-1337

Greene D 2002 JVS, alliances, consortia on path to survival for many, Semiconductor Magazine, June 2002

Haeringer G 2001 On the stability of cooperation structures, Working Paper, Department of Economics, Warwick University, Coventry, U.K. 1-19

Handfield R B, Bechtel C 2002 The role of trust and relationship structure in improving supply chain responsiveness. Industrial Marketing Management 31(4): 367-382

Hartman B C, Dror M 1996 Cost allocation in continuous review inventory models. Naval Research Logistics 43: 549-561

Leng M, Parlar M 2005 Allocation of cost savings in a three-level supply chain with demand information sharing: A cooperative-game approach, Working Paper, McMaster University, Hamilton, ON

Littlechild S C, Owen G 1973 A simple expression for the Shapely value in a special case. Management Science 20: 370-372

Mas-Colell A, Whinston M D, Green J R 1995 Microeconomic theory, (Oxford, U.K: Oxford University Press)

Meca A, Timmer J, Garcia-Jurado I, Borm P E M 2004 Inventory games. European J. Operational Res. 156: 127-139

Moulin H 1988 Axioms of Cooperative decision making, (Cambridge, U.K: Cambridge University Press)

Myersom R B 1977 Graphs and cooperation in games. Mathematical Operation Res. 2: 225-229

Myerson R 1991 Game theory: Analysis of conflict, (Cambridge, MA: Harvard University press)

Nagarajan M, Sosic G 2006 Game-theoretic analysis of cooperation among supply chain agents: Review and extensions. European J. Operational Res. 187(3) 719-745

Park S, Suresh N C 2005 An investigation of the roles of electronic marketplaces in the supply chains, Proceedings of IEEE $38^{\text {th }}$ Hawaii International Conference on System Sciences, 1-10

Plambeck E L, Taylor T A 2004 Implications of breach remedy and renegotiation for design of supply contracts, Working paper, (New York, NY: Columbia University)

Premkumar G P 2003 Perspectives of e-marketplace by multiple stake holders. Communications of ACM 46(12): 279-288

Qin C-Z 1996 Endogenous formation of cooperation structures. J. Economic Theory 69: 218-226

Raghunathan S 2003 Impact of demand correlation in the value of and incentives for information sharing in a supply chain. European J. Operational Res. 146: 634-649

Reinhardt G, Dada M 2005 Allocating the gains from resource pooling with the Shapley value. J. Operational Res. Soc. 56: $997-1000$

Roth A E 1988 Introduction to the shapely value, in The Shapely Value: Essays in honor of Lloyed S. Shapely, Cambridge University Press, 1-27

Sauvee L 1998 Towards an institutional analysis of vertical coordination in agribusiness, in the Industrialization of Agriculture, J Royer, R Rojers (eds.) Aldershot, England, Ashgate Publishing Co.

Schmeidler D 1969 The nucleolus of a characteristic function game. SIAM J. Applied Mathematics 17: $1163-1170$

Shapley L S 1953 A value for N-Person games, In: Contribution to the Theory of Games, vol. 2 (Princeton, NJ: Princeton University Press) 307-317

Subramanian C, Shaw M J 2004 The effects of process characteristics on the value of B2B e-procurement. Information Technology and Management 5(1-2): 161-180 